\title{
REPRODUCIBILITY OF SCHATZKER CLASSIFICATION THROUGH SMARTPHONE APPLICATIONS
}

\author{
Mauro Rodrigues dos Santos ${ }^{1}$, Junichiro Sado Júnior ${ }^{1}$, Roberto Medeiros de Sousa ${ }^{1}$, Osman Rodrigues Roriz ${ }^{1}$ \\ 1. Universidade Federal de Goiás, Hospital das Clínicas, Department of Orthopedics and Traumatology, Knee Group, Goiânia, GO, Brazil.
}

\section{ABSTRACT}

Objective: To evaluate the intra-observer reproducibility of Schatzker classification for tibial plateau fractures through smartphone applications. Methods: Radiographs were evaluated in two incidences (anteroposterior and profile) and CT slices (axial, sagittal and coronal) of 37 patients with tibial plateau fracture. Two evaluators, knee surgery experts, classified the cases by viewing the images of the isolated radiographs and then $\mathrm{X}$-rays associated with CT slices in four different stages via smartphones and then presential assessment. Data were statistically analyzed with the Kappa coefficient (k). Results: There was intra-observer agreement by comparing the two methods of evaluation: display or via smartphone, and the analysis made showed statistical significance. Conclusion: The use of smartphones did not affect the reliability of Schatzker classification. Level of Evidence III, Diagnostic Study - Investigating a Diagnostic Test.

Keywords: Tibial fractures/classification. Tibial fractures/radiography. Cell phones/utilization. Mobile applications/utilization.

Citation: Santos MR, Sado Júnior J, Sousa RM, Roriz OR. Reproducibility of Schatzker classification through smartphone applications. Acta Ortop Bras. [online]. 2016;24(6):309-11. Available from URL: http://www.scielo.br/aob.

\section{INTRODUCTION}

Tibial plateau fractures are common injuries that include a wide range of morphological patterns. A number of classification systems have been proposed to categorize these types of fractures, in order to simplify communication in clinical practice, to provide guidelines for preoperative planning, and to allow comparison with the data presented in the literature. The three classification systems used in the assessment of tibial plateau fractures include the OTA/AO system, the Schatzker classification and Hohl's classification. ${ }^{1-3}$

$X$-rays images and CT scans are commonly and frequently shared through smartphone devices between orthopedic surgeons and fellow residents, a technological facility that allows the debate at any time with team members who are far away from each other. Obviously, it does not replace the presential assessment and care provided to the patient by a qualified professional.

Currently, there are numerous software applications that can be installed on smartphones. Health-related applications are increasingly being developed, with approximately 1,000 new applications launched every month and 142 million downloads annually, estimated by 2016 . Its use is popular, with $85 \%$ of medical professionals using smartphones and $30-50 \%$ using applications in clinical practice. The range of applications available for smartphones has been reported in various specialties, including orthopedics, colorectal surgery, anesthesiology, radiology and microbiology. ${ }^{4-9}$ However, concerns regarding the lack of physician's involvement in developing the application and the reliability of the application's content have been raised.

The use of smartphones applications in orthopedics have been described in the literature, such as measuring the angular deviation in hallux valgus ${ }^{4,5}$ and spinal deformities, ${ }^{6}$ measuring the flexion-extension after arthroplasty ${ }^{9}$ and also the use of these applications in radiology. $8,10-12$

The aim of this study is to evaluate the intra-observer reliability of the Schatzker classification for tibial plateau fractures using smartphones.

\section{MATERIALS AND METHODS}

We retrospectively evaluated 37 cases of tibial plateau fractures treated at the same hospital between 2011 and 2014. All patients had knee radiographs in anteroposterior (AP) and lateral (L) views supplemented with computed tomography (CT). All these tests were stored in digital media.

The two evaluators were orthopedic surgeons, members of the Brazilian Society of Orthopedics and Traumatology (SBOT) and the Brazilian Society of Knee Surgery (SBCJ) with expertise in the treatment of tibial plateau fractures. They will be hereafter designated as evaluator 1 and evaluator 2 .

In the first stage, all AP and $L$ radiographs were photographed using a smartphone and sent, case by case, in random and independent order to smartphones of the two evaluators using

All the authors declare that there is no potential conflict of interest referring to this article. 
the mobile application Whatsapp ${ }^{\circledR}$. Then, they applied the Schatzker classification and sent the evaluations to the examiner, which, in turn, sent the photos of other cases to the evaluators, successively, until completion of the 37 cases.

The second stage was carried out similarly to the first, in random and independent order, however, in addition to the X-rays, CT images in coronal, axial and sagittal views were added to each evaluated case.

In the third and fourth steps, performed six months after the first, the same cases and images were rated by two evaluators in presential way, directly from the hospital's computer monitor. In the third stage, the examiner presented only the X-ray image, and in the fourth stage, X-rays and CT scans.

The smartphones used for both capturing and sending images and classifying images by the evaluators were Apple ${ }^{\circledR}$ iPhone $5 S$ model with 4-inch screen. Regarding the presential assessment, the images were displayed on a full HD 21.5-inch monitor (1920 $x 1080$ pixels), and the software DViewer was used to visualize the images. Both the computer as the smartphone software allowed magnification of the image on their screens.

Data were entered and manipulated in Excel charts, for further processing using the Statistical Package for Social Sciences (SPSS) for Windows (version 21.0).

For concordance analysis was used the Kappa test. The values of this index ranged from +1 (perfect agreement) through zero (fortuitous or chance agreement) up to -1 (no agreement). In general, values lower than 0.5 were considered unsatisfactory, values between 0.5 and 0.75 were satisfactory, and values higher than 0.75 are considered excelent. ${ }^{13}$

For all tests we used a 95\% level of confidence, i.e., $p<0.05$ was considered statistically significant. This study, that dealt with analysis of medical records, did not require approval by the Ethics Committee.

\section{RESULTS}

Both evaluators showed significant agreement, with Kappa above 0.7 and $p<0.001$, in X-ray only presential and smartphone intra-observer evaluations. (Table 1)

Regarding presential and smartphone evaluation of X-ray image and CT scan, we observed that despite the evaluator 1 showed Kappa 0.68 and the evaluator 2 Kappa 0.81, both had very good and significant agreement with Kappa above 0.6 and $p<0.001$. (Table 2)

The analysis of observations together by both evaluators, both presential as smartphone evaluations of $\mathrm{x}$-ray alone or together with CT scan showed significant agreement, with Kappa above 0.7 and $p<0.001$. (Table 3)

\section{DISCUSSION}

A common and frequent practice among orthopedic surgeons and residents is to share radiological images and tomography scans by electronic means. However, this practice requires scientific validation, since it is not known whether this technology may lead to mistaken interpretation of the images, and, consequently, improper decision making by the surgical team.
Table 1. Concordance analysis of the results of X-ray observations in presential way and using smartphones.

\begin{tabular}{c|c|c|c|c|c|c}
\hline \multirow{2}{*}{ Observer } & \multicolumn{2}{|c|}{ Concordance } & \multicolumn{2}{|c|}{ Discordance } & \multirow{2}{*}{ Kappa } & $\boldsymbol{p}$ \\
\cline { 2 - 5 } & $\mathbf{N}$ & $\%$ & $\mathbf{n}$ & $\%$ & & \\
\hline Evaluator 1 & 30 & 81.1 & 7 & 18.9 & 0.77 & $<0.001$ \\
\hline Evaluator 2 & 29 & 78.4 & 8 & 21.6 & 0.73 & $<0.001$ \\
\hline
\end{tabular}

\begin{tabular}{|c|c|c|c|c|c|c|}
\hline \multirow{2}{*}{ Observer } & \multicolumn{2}{|c|}{ Concordance } & \multicolumn{2}{|c|}{ Discordance } & \multirow{2}{*}{ Kappa } & \multirow{2}{*}{$p$} \\
\hline & N & $\%$ & $\mathbf{n}$ & $\%$ & & \\
\hline Evaluator 1 & 28 & 75.7 & 9 & 24.3 & 0.68 & $<0.001$ \\
\hline Evaluator 2 & 32 & 86.5 & 5 & 13.5 & 0.81 & $<0.001$ \\
\hline
\end{tabular}

Table 3. Concordance analysis of the results of observations by evaluator $1+$ evaluator 2 in presential way and using smartphones.

\begin{tabular}{c|c|c|c|c|c|c}
\hline \multirow{2}{*}{ Method } & \multicolumn{2}{|c|}{ Concordance } & \multicolumn{2}{|c|}{ Discordance } & \multirow{2}{*}{ Kappa } & \multirow{p}{*}{} \\
\cline { 2 - 5 } & $\mathbf{N}$ & $\%$ & $\mathbf{n}$ & $\%$ & & \\
\hline $\mathrm{RX}$ & 59 & 79.7 & 15 & 20.3 & 0.75 & $<0.001$ \\
\hline $\mathrm{RX}+\mathrm{TC}$ & 60 & 81.1 & 14 & 18.9 & 0.75 & $<0.001$ \\
\hline
\end{tabular}

In this study, the application of Schatzker classification for tibial plateau fractures through images interpreted on smartphones screens versus presential screening in intra-observer analysis was considered satisfactory or excellent, with all indices above 0.5 , the largest 0.81 and the lowest 0.68 .

It should be noticed that the two evaluators are orthopedic surgeons experienced in the treatment of tibial plateau fractures, which enhanced the degree of agreement. In a study by Albuquerque et al., ${ }^{14}$ it has been shown that inexperienced surgeons tend to have lower intra- and inter-observer variability for the different classifications of tibial plateau fractures (Hohl, AO or Schatzker). Therefore, we chose to employ experienced surgeons in this work, minimizing the effects of this important variable, enabling us to focus specifically on possible influences of smartphone devices on the results.

Other studies have observed unsatisfactory K indices (near 0.38) in inter-observer analysis ${ }^{2,15}$ and satisfactory kappa values (0.68) in intra-observer analysis ${ }^{2}$ for this classification, which makes clear that the correlation value decreases when the evaluation is between the evaluators. In this study, the concordance results are similar to results obtained in intra-observer analysis for Schatzker classification.

\section{CONCLUSION}

The use of images through smartphone devices did not interfere with the intra-observer agreement of Schatzker classification of tibial plateau fractures in this study. The same result was obtained to rank $\mathrm{X}$-rays only and also $\mathrm{X}$-rays combined with tomography scans. 
AUTHORS' CONTRIBUTIONS: Each author contributed individually and significantly to the development of the study. RMS (0000-0002-31768608)* and ORR (0000-0002-1857-6628)* were the main contributors in drafting the manuscript and literature review. MRS (0000-0002-1339$5801)^{\star}$ and JSJ (0000-0002-5362-5579)* were the creators, advisors and the two evaluators who performed the classification of images of fractures. RMS and JSJ evaluated the statistical data collected. All authors contributed to the final review of the manuscript. ${ }^{\star}$ ORCID (Open Researcher and Contributor ID).

\section{REFERENCES}

1. Brunner A, Horisberger M, Ulmar B, Hoffmann A, Babst R. Classification systems for tibial plateau fractures; does computed tomography scanning improve their reliability? Injury. 2010;41(2):173-8.

2. Walton NP, Harish S, Roberts C, Blundell C. AO or Schatzker? How reliable is classification of tibial plateau fractures? Arch Orthop Trauma Surg. 2003;123(8):396-8.

3. Maripuri SN, Rao P, Manoj-Thomas A, Mohanty K. The classification systems for tibial plateau fractures: how reliable are they? Injury. 2008;39(10):1216-21.

4. Walter R, Kosy JD, Cove R. Inter- and intra-observer reliability of a smartphone application for measuring hallux valgus angles. Foot Ankle Surg. 2013;19(1):18-21.

5. Ege T, Kose O, Koca K, Demiralp B, Basbozkurt M. Use of the iPhone for radiographic evaluation of hallux valgus. Skeletal Radiol. 2013;42(2):269-73.

6. Jacquot F, Charpentier A, Khelifi S, Gastambide D, Rigal R, Sautet A. Measuring the Cobb angle with the iPhone in kyphoses: a reliability study. Int Orthop. 2012;36(8):1655-60.

7. Franko OI. Smartphone apps for orthopaedic surgeons. Clin Orthop Relat Res. 2011;469(7):2042-8.

8. McNulty JP, Ryan JT, Evanoff MG, Rainford LA. Flexible image evaluation: iPad versus secondary-class monitors for review of MR spinal emergency cases, a comparative study. Acad Radiol. 2012;19(8):1023-8.
9. Jenny JY. Measurement of the knee flexion angle with a Smartphone-application is precise and accurate. J Arthroplasty. 2013;28(5):784-7.

10. Rodrigues MA, Visvanathan A, Murchison JT, Brady RR. Radiology smartphone applications; current provision and cautions. Insights Imaging. 2013;4(5):555-62.

11. John S, Poh AC, Lim TC, Chan EH, Chong le R. The iPad tablet computer for mobile on-call radiology diagnosis? Auditing discrepancy in CT and MRI reporting. J Digit Imaging. 2012;25(5):628-34.

12. Székely A, Talanow R, Bágyi P. Smartphones, tablets and mobile applications for radiology. Eur J Radiol. 2013;82(5):829-36.

13. Dirchl DR, Cannada LK. Classification of fractures. In: Bucholz RW, Heckman JD, Court-Brown Cm, Torneta P 3rd, McQueen MM, Ricci WM, editors. Rockwood \& Grenn fractures in adults. 7th ed. Philadelphia: Lippincott Williams \& Wilkins; 2009. p. 45-51.

14. Albuquerque RP, Giordano V, Pallottino A, Sassine T, Canedo R, Pina J, et al Analysis of the reproducibility of tibial plateau fractures' classification. Rev Bras Ortop. 2015;16;44(3):225-9.

15. de Lima Lopes C, da Rocha Cândido Filho CA, de Lima E Silva TA, Gonçalves MC, de Oliveira RL, de Lima PR. Importance of radiological studies by means of computed tomography for managing fractures of the tibial plateau. Rev Bras Ortop. 2014;30;49(6):593-601. 